\title{
Age-Related Changes in Bone Mineral Density, Cross-Sectional Area and Strength at Different Skeletal Sites in Male Rats
}

\author{
Haruzo IIDA $^{1)}$ and Satoshi FUKUDA ${ }^{1)}$ \\ ${ }^{1)}$ International Space Radiation Laboratory, National Institute of Radiological Sciences, 4-9-1 Anagawa, Inage-ku, Chiba-shi, Chiba 263- \\ 8555, Japan
}

(Received 30 January 2001/Accepted 4 September 2001)

ABSTRACT. Age-related changes in bone mineral density (BMD), cross-sectional area and strength strain index (SSI) of the long bones in the limbs and first lumbar vertebra of male Wistar rats were measured by a peripheral quantitative computed tomography (pQCT) method. One hundred and ten rats aged 2-30 months were used. The results indicate that the total (cortical + trabecular), cortical and trabecular BMD values of the metaphysis and cortical BMD values of the diaphysis in the long bones varied for each bone and differed from those of the first lumbar vertebra. The total BMD of long bones showed high values at 6-21 months and then decreased, but these did not always coincide with cortical and trabecular BMD. The values of SSI in the long bones varied. The values of total and cortical BMD and SSI of lumbar vertebra increased for 6-12 months and then decreased, but the trabecular BMD increased after 12 months. The total area in both the long bones and the first lumbar vertebra increased with the decrease in cortical area and the increase in the trabecular area with increasing age. It was concluded that age-related changes in bones, similar to those observed in humans, could be observed in some bones and parameters, although the age in rats when the so-called peak bone mass appears in the whole skeleton could not be clearly determined.

KEY WORDS: age change, bone mineral density, male rat, peak bone mass, peripheral quantitative computed tomography (pQCT)

J. Vet. Med. Sci. 64(1): 29-34, 2002

Rats are widely used for studies on bone metabolism and diseases such as osteoporosis, although their worth as an animal model has been contested $[6,12,16,21]$ because their bone morphological patterns differ from those of humans. Rats show a modeling pattern from youth to old age, while humans show a modeling pattern in the growth stage which changes to a remodeling pattern after maturity [7]. The length and weight of long bones in rats continue to increase up to the old age due to the fact that both or either of the epiphysial growth plates do not close for a long time [8]. The cortical and trabecular bone components of the femur neck in rats have been found to be higher and lower, respectively, than those in humans [3].

The age of peak bone mass, hereafter referred to as bone mineral density, in a human is considered a significant indicator for knowing the state of activity of bone metabolism and age of onset of osteoporosis, because thereafter the bone mass reduces with increasing age. To clarify whether rats show peak bone mass or not is an important matter for considering their worth as a model animal in bone researches and for determining corresponding ages between rats and humans in experimental procedures and assessing experimental results. That is, we are interested to know if rats have age-related changes in bone metabolism throughout their life similar to those in humans. We demonstrated that high values of mineral and organic contents at various skeletal sites are present in rats [11] and a peak of bone volume in the proximal metaphysis of the tibia is revealed by means of histomorphometry [9]. Many researchers have observed age-related changes in bone mineral density and morphology, but the results have been varied. Probably, this is due to differences in small numbers of observed sites and differ- ent measurement methods [2-4, 13, 18-20].

Some technologies, such as quantitative computed tomography (QCT), dual energy x-ray absorptiometry (DEXA), and CT microscope (micro-CT) are available to measure bone properties such as bone mineral density and content. QCT technology offers many opportunities for the investigation of bone bio-mechanics because it determines some indicators of bone properties that are relevant to bone strength, bone mass, and the mechanical quality such as bone marrow and cortical bone areas. The property of QCT is to separate the faster reacting cancellous compartment from the more slowly reacting but mechanically more important cortical compartment after stimulation of mechanical stress and administration of drugs. DEXA cannot separate the cancellous and cortical compartments, and Micro-CT is able to analyze 3-D histomorphometrical images of trabecular bone specimens but cannot determine bone density values. The disadvantage of Micro-CT is its high cost and the long determination times needed. Because the peripheral QCT (pQCT) was developed as a small-field, high-resolution extension of the existing QCT system, it is available for animal studies as well as for clinic use for humans.

The purpose of the present study was to clarify the characteristics of age-related changes in bone properties such as BMD, cross-section area and strength in different skeletal sites in rats, particularly whether peak of BMD presents in male rats or not.

\section{MATERIALS AND METHODS}

A total of one hundred and ten male Wistar Mishima 
(WM/Nrs) rats were used. Ten rats were sacrificed at the respective ages of $2,3,6,9,12,15,18,21,24,27$ and 30 months. The mean \pm standard deviation of life span of this strain in our facility is $725 \pm 176$ days $(n=918)$. The ages at the survival rates of $90 \%, 50 \%$ and $10 \%$ were 514,744 and 915 days after birth, respectively. Rats were housed five to a stainless steel cage under conventional conditions. They were kept in an animal room controlled at a constant condition (temperature: $22 \pm 2{ }^{\circ} \mathrm{C}$; relative humidity, $55 \pm 5 \% ; 12$ hr light-dark cycle). They were fed a commercial diet (MB1, Funabashi Farm Co. Ltd., Japan) containing 1.0\% calcium, $1.2 \%$ phosphorus and water ad libitum.

Under anesthesia induced by chloroform inhalation, the body weight was measured, and four bones (femur, tibia, humerus and first lumbar vertebra) were extracted and the muscles and tendons were removed.

The experimental animals used in this study were treated and/or handled according to the "Recommendations for the Handling of Laboratory Animals for Biomedical Research", compiled by the Committee for the Safety and Ethical Handling Regulations for Laboratory Animal Experiments at our institute.

Measurements of BMD and cross-sectional area were performed using a pQCT (Norland \& Stratec XCT-960A). In this system, values of less than 395 and more than 690 $\mathrm{mg} / \mathrm{cm}^{3}$ are regarded as trabecular BMD and cortical BMD, respectively. The strength strain index (SSI) was also calculated by software [10].

SSI $=$ pCSMI. $\times$ Cortical vBMDi $/ \mathrm{dMx} . \times$ vBMDMx, where pCSMI is relevant to long bone stiffness and strength in bending and torsion. pCSMI $=\sum\left(\mathrm{A}_{\mathrm{i}} \cdot \mathrm{d}_{\mathrm{i}}{ }^{2}\right)$, where $\mathrm{Ai}$ is the area of an individual voxel within the bone section; di is the distance from the center of that area to the reference, bending $(\mathrm{x}, \mathrm{y})$ or torsion $(\mathrm{z})$ axis. $\mathrm{dMx}$ is the maximal distance from a voxel to the polar $\mathrm{z}$ axis in the image, and vBMFDMx is the maximal value the cortical vBMD could theoretically assume $\left(464 \mathrm{mg} / \mathrm{cm}^{3}\right)$.

Total (cortical + trabecular), cortical and trabecular $\mathrm{BMD}$, and cross-sectional area in the metaphysis, and cortical BMD and cross-sectional area in the diaphysis of long bones were measured at 3 and $12 \mathrm{~mm}$ from the growth plate in the distal epiphysis of the femur and the proximal epiphysis of the tibia and humerus, respectively. The measurement of the first lumbar vertebra was performed at the center of the transverse direction.

Statistical analyses were performed by one-way ANOVA test and Student' $t$ test. The value with no significant differences $(p<0.05)$ in the ranges showing high values was regarded as a high value.

\section{RESULTS}

Body weight (Fig. 1): The body weights of rats increased until they were 12 months old, did not change between 12 and 24 months old, and then decreased.

BMD values in the metaphysis and diaphysis of long bones (Figs. 2 and Fig. 3): The total BMD was high at 6-21

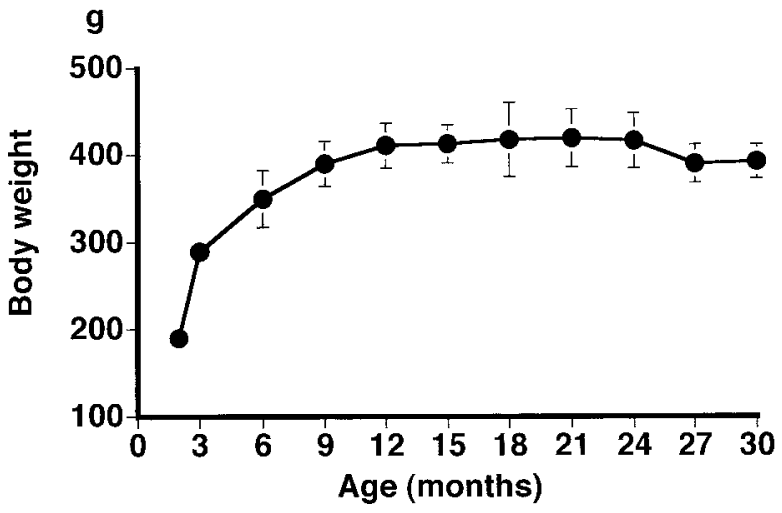

Fig. 1. Changes in body weight of rats. Values and bar are mean \pm SD.

months in the femur and tibia, and 9-21 months in the humerus (Fig. 2a). The cortical BMD was high at 6-30 (except 15) months in the femur, 6-30 months in the tibia, and 9-30 (except 24) months in the humerus (Fig. 2b). The trabecular BMD was high at 12-30 months in the femur, 921 month in the tibia, and 3-30 months in the humerus (Fig. 2c). The cortical BMD of the diaphysis was high at 9-18 months in the femur, at 12-24 months in the tibia, and at 224 months in the humerus (Fig. 3).

$B M D$ in the center of first lumbar vertebra (Figs. 4): In the first lumbar vertebra, the total, cortical and trabecular BMD were high at 6-12 (Fig. 4a), 3-12 (Fig. 4b) and 15-30 months (Fig. 4c), respectively.

Cross-sectional area of each bone (Figs. 5): In the distal metaphysis of the femur, the total area was high at 9-30 months, and the trabecular area did not change between 221 months but thereafter increased. The cortical area increased until 9 months, did not change between 9-18 months, and then decreased (Fig. 5a). In the proximal metaphysis of the tibia, the total area increased until 9 months and thereafter tended to continuously increase but not significantly, and the trabecular area did not change between 2-18 months but then increased. The cortical area increased until 9 months, was high between 9-21 months and then deceased (Fig. 5b). In the proximal metaphysis of the humerus, the total area increased from 2 months until 30 months, but did not change significantly between 12-21 months. The trabecular area did not change between 2-21 months, but increased after 24 months. The cortical area increased until 12 months, but thereafter did not significantly change (Fig. 5c).

The cortical area of the diaphysis of the femur increased with increasing age until 12 months, did not change between 12-24 months, and then increased. That of the tibia increased until 9 months, did not change between 9-21 months, and then decreased. That of the humerus increased until 9 months, and then did not change (Fig. 5d).

In the central cross-section of the first lumbar vertebra, the total area increased until 15 months and then did not sig- 

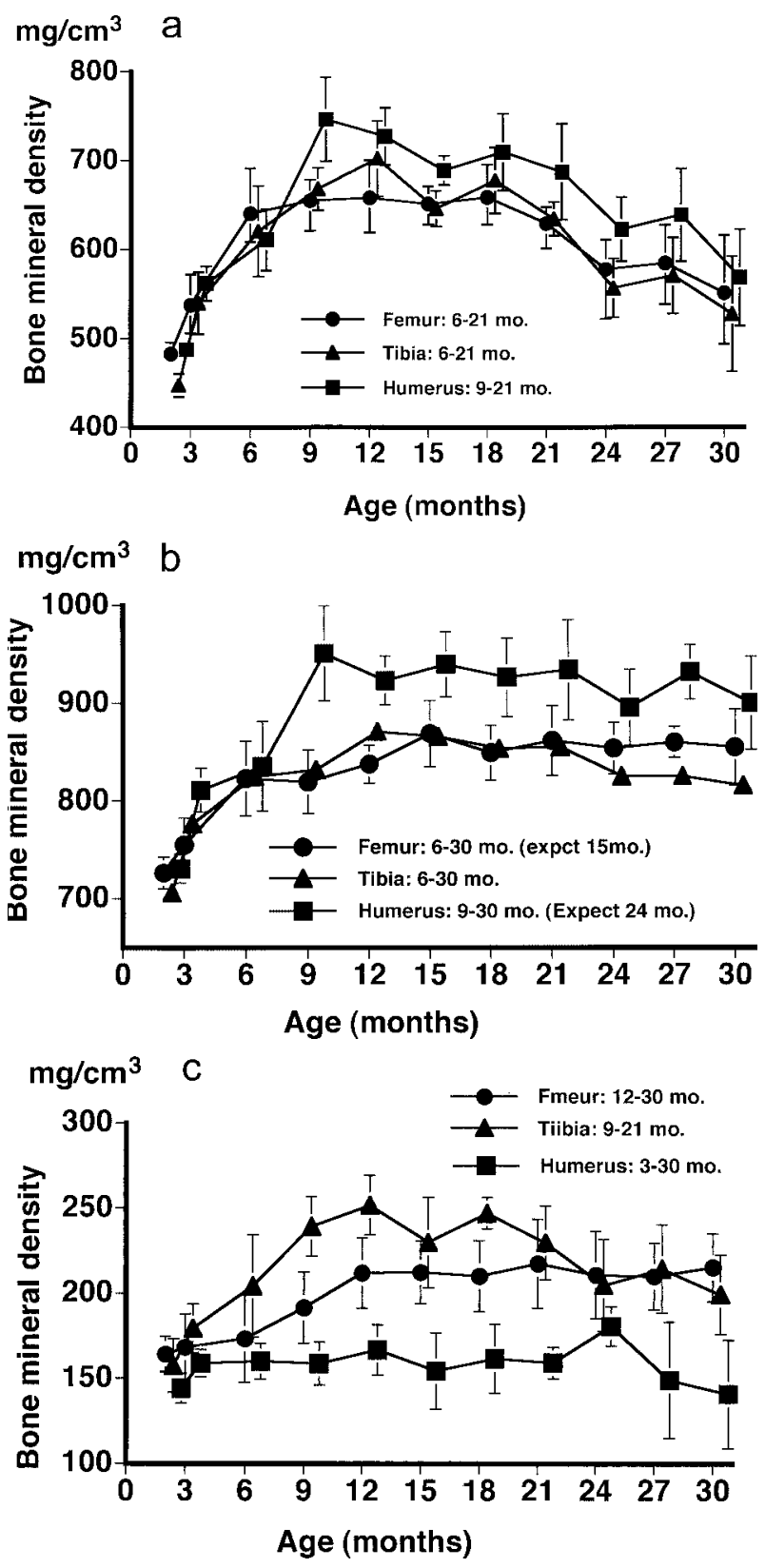

Fig. 2. Total (a), trabecular (b) and cortical (c) BMD values in the metaphysis of femur, tibia and humerus. The ages showing high values are presented in each figure. Values and bar are mean $\pm \mathrm{SD}$.

nificantly change. The trabecular area did not change between $2-9$ months, and thereafter increased. The cortical area did not change between 6-21 months and thereafter decreased (Fig. 6).

SSI of the long bone and first lumbar vertebra (Fig. 7): The SSI of the metaphysis of the femur increased until 9 months and then did not change. The value of the tibia did not change between 12-27 months, and that of the humerus did not change between 12-30 months (Fig. 7a). The SSI of

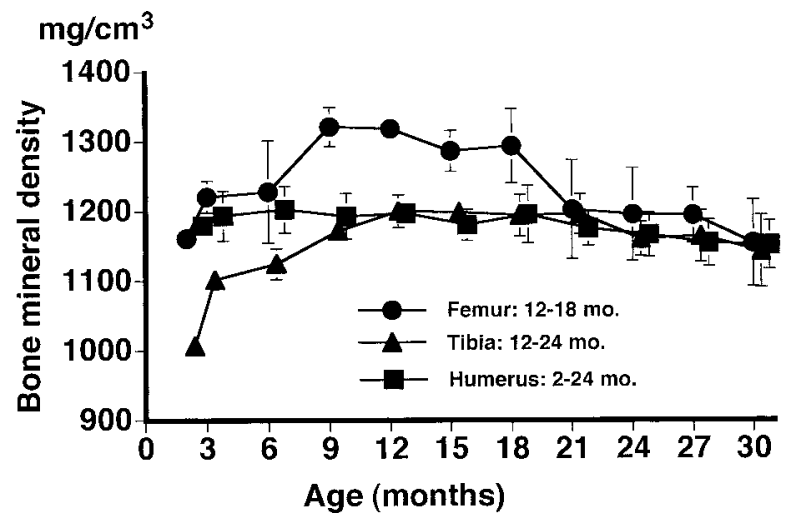

Fig. 3. Cortical BMD values in the diaphyses of femur, tibia and humerus. Values are mean \pm SD. The ages showing high values are presented in the figure.

the diaphysis of femur increased until 9 months, and then continued to increase until 18 months after which it did not change. Those of the tibia and humerus increased until 12 months and then did not change (Fig. 7b). The SSI of the first lumbar vertebra was high between 9-21 months and then decreased (Fig. 7c).

\section{DISCUSSION}

The total BMD in the metaphyses of femur, tibia, humerus and the center of the transverse direction of the first lumbar vertebra increased to reach high values, and thereafter decreased with increasing age. The age-related changes in the total BMD of all bones were similar to those of humans. However, differences were observed between long bones and first lumbar vertebra, also among long bones at different sites of the same bone.

In the long bones, the period showing high values of total BMD in the metaphysis of the humerus in the fore-limb was between 9-12 months and those of the femur and tibia in the hind-limb were between 6-21 months. The difference in the periods between fore- and hind-limbs may be related to the burden of body weight, because the burden ratio of the body weight shared by the fore and hind limbs is $40: 60 \%$, when a rat stands. Compared to the changes in the cortical and trabecular BMD among the long bones after they reached the high values, the values in the femur and humerus did not change, while those of the tibia decreased. This agrees with the results on the changes in calcium content in the femur and tibia of our previous study [11]. This may be affected by the different times when the growth plate is closed: at 15-17 weeks in the distal epiphysis of the femur, more than 104 weeks in the proximal epiphysis of the tibia, and 52 weeks in the distal epiphysis of the humerus [8].

The difference of interest is that although the total BMD decreased, both the cortical and trabecular BMD did not change in the femur and humerus. The reason for this might be an increase in sub-cortical bone such as the end-surface and periosteal parts, identified by threshold values between 

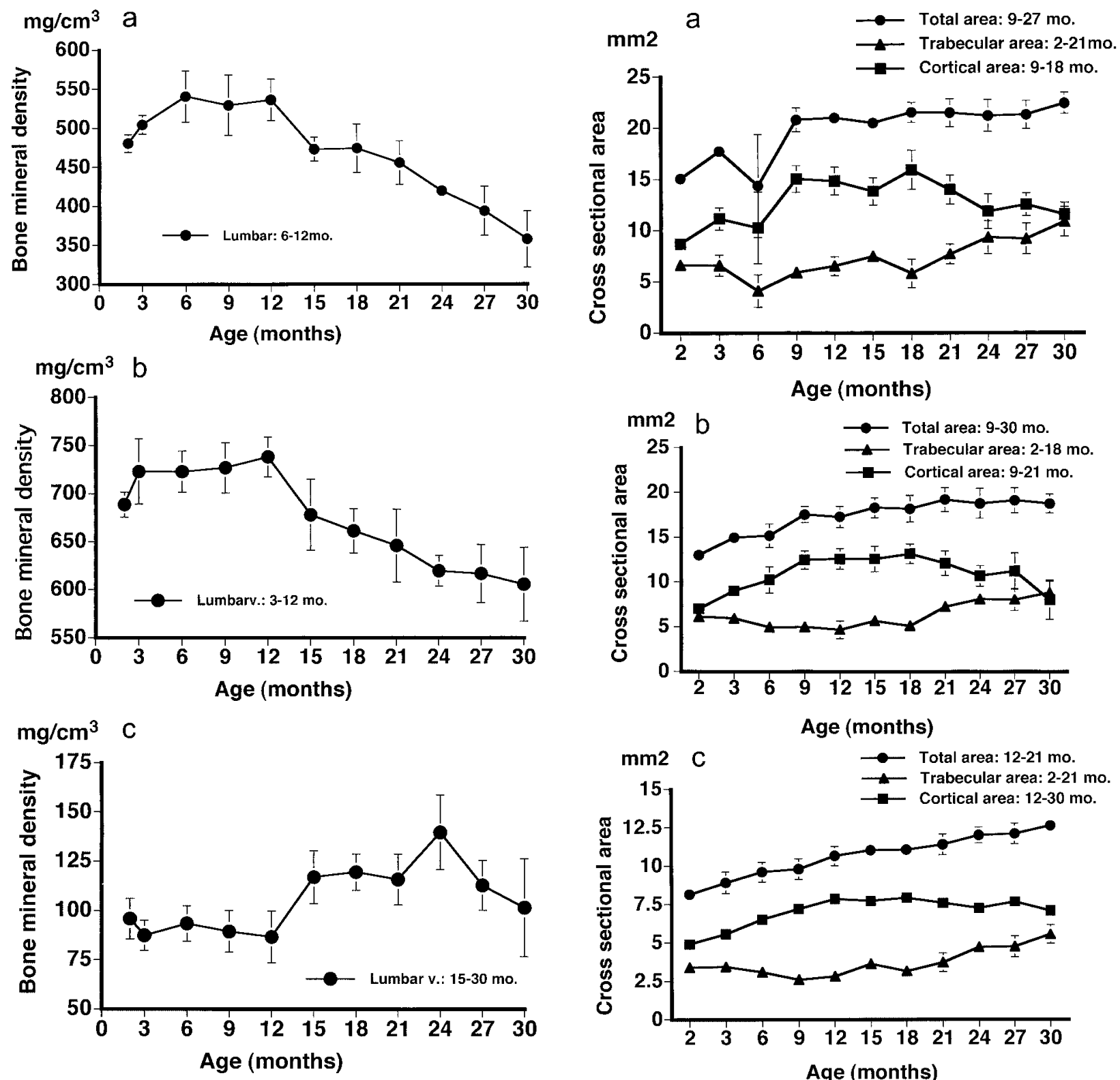

Fig. 4. Total (a), trabecular (b) and cortical (c) BMD values in the first lumbar vertebra. The ages showing high values are presented in each figure. Values and bar are mean \pm SD.

395 and $690 \mathrm{mg} / \mathrm{cm}^{3}$ accompanied by morphological changes. The data on area obtained in this study indicated that, the trabecular area increased while the cortical area decreased, suggesting that the sub-cortical area increased with increasing age. That is, the age-related changes in the

Fig. 5. Cross-sectional areas in the metaphyses of femur (a), tibia (b), humerus (c), and diaphyses of long bones (d). Values are mean \pm SD. The ages showing high values are presented in each figure. Values and bar are mean \pm SD.
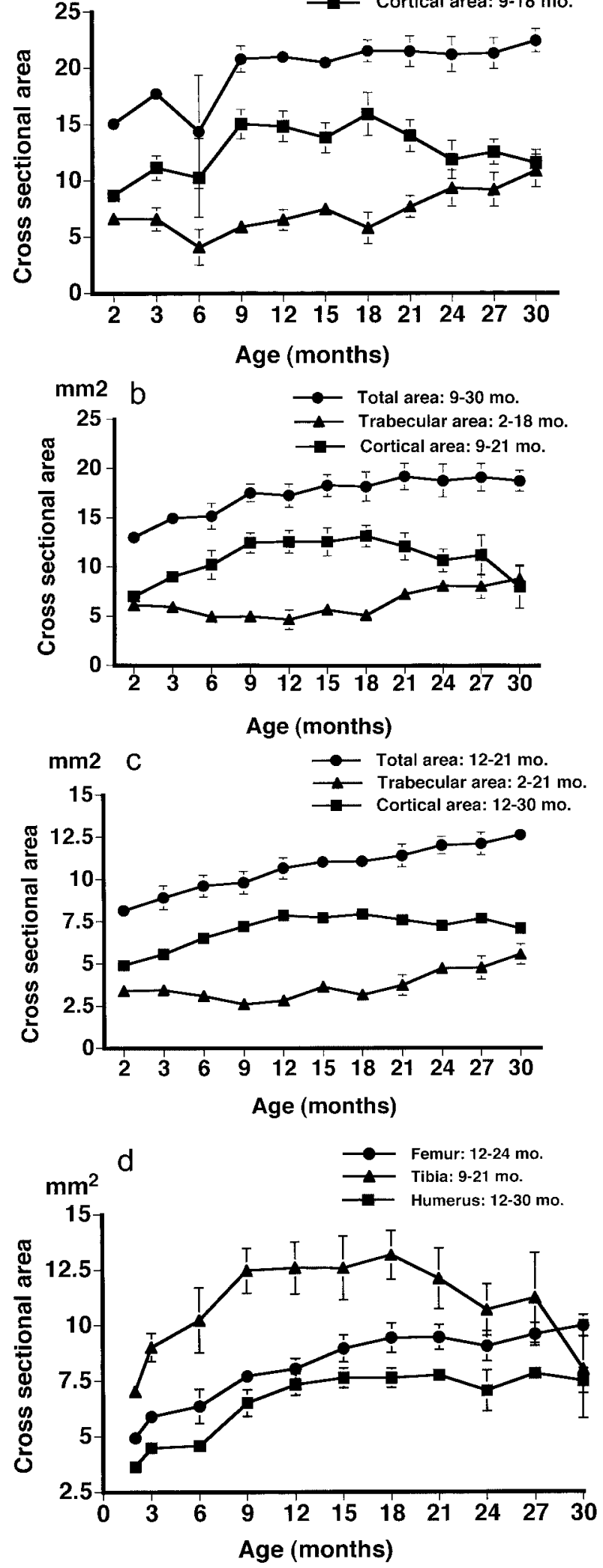


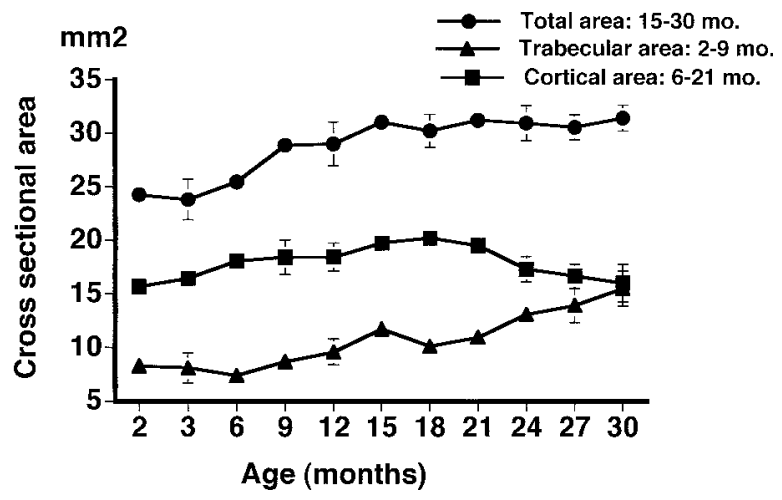

Fig. 6. Cross-sectional area in first lumbar vertebra. The ages showing high values are presented in the figure. Values and bar are mean \pm SD.

total BMD in the metaphysis of long bones is due to morphological change in the sub-cortical area, and is not accompanied with decreases in cortical and trabecular BMD.

In the first lumbar vertebra, the period showing high value of total BMD was 6-12 months after which is decreased. This corresponds with previous results on the calcium content in the first lumbar vertebra [11]. However, the cortical BMD revealed a pattern similar to that of total BMD, although the trabecular BMD did not change until 12 months and then increased. In all cases, the decrease in the total BMD in the first lumbar vertebra with increasing age is attributed to the change in cortical BMD which occupies a high percent of total BMD, in addition to the morphological changes, because it was distinctly observed that the cortical area decreased but the trabecular area increased compared with those of long bones.

Much evidence has shown that BMD decreases with increasing age in men, and is accompanied by decreasing sex hormones, but more moderately than in post-menopausal women $[5,10,17,18,23,24]$. According to previous studies, the high BMD or calcium content of the first and fourth lumbar vertebrae, femur, and tibia coincided with a high testosterone level at the age of 12 months in male Wistar rats $[11,14,17]$. On the other hand, no change in the BMD of the femur measured by DEXA in male rats was observed after the rats had reached maturity [19]. Therefore, although sex hormones act to increase or maintain BMD level, the concentration required for bone metabolism may not be as high as that needed for reproduction and growth $[1,15,17-18,22,24]$.

Together with the data obtained from the present and previous studies, the age when highest total BMD in the whole skeleton of male Wistar rats occurs is suggested to be around the age of 12 months, although the high values ranged between 6 and 21 months. After 12 months old, the total BMD decreased in the first lumbar and tibia, but did not change in the femur and humerus. Therefore, the socalled peak of BMD was observed in the first lumbar vertebra and tibia, but not in the femur and humerus. This result
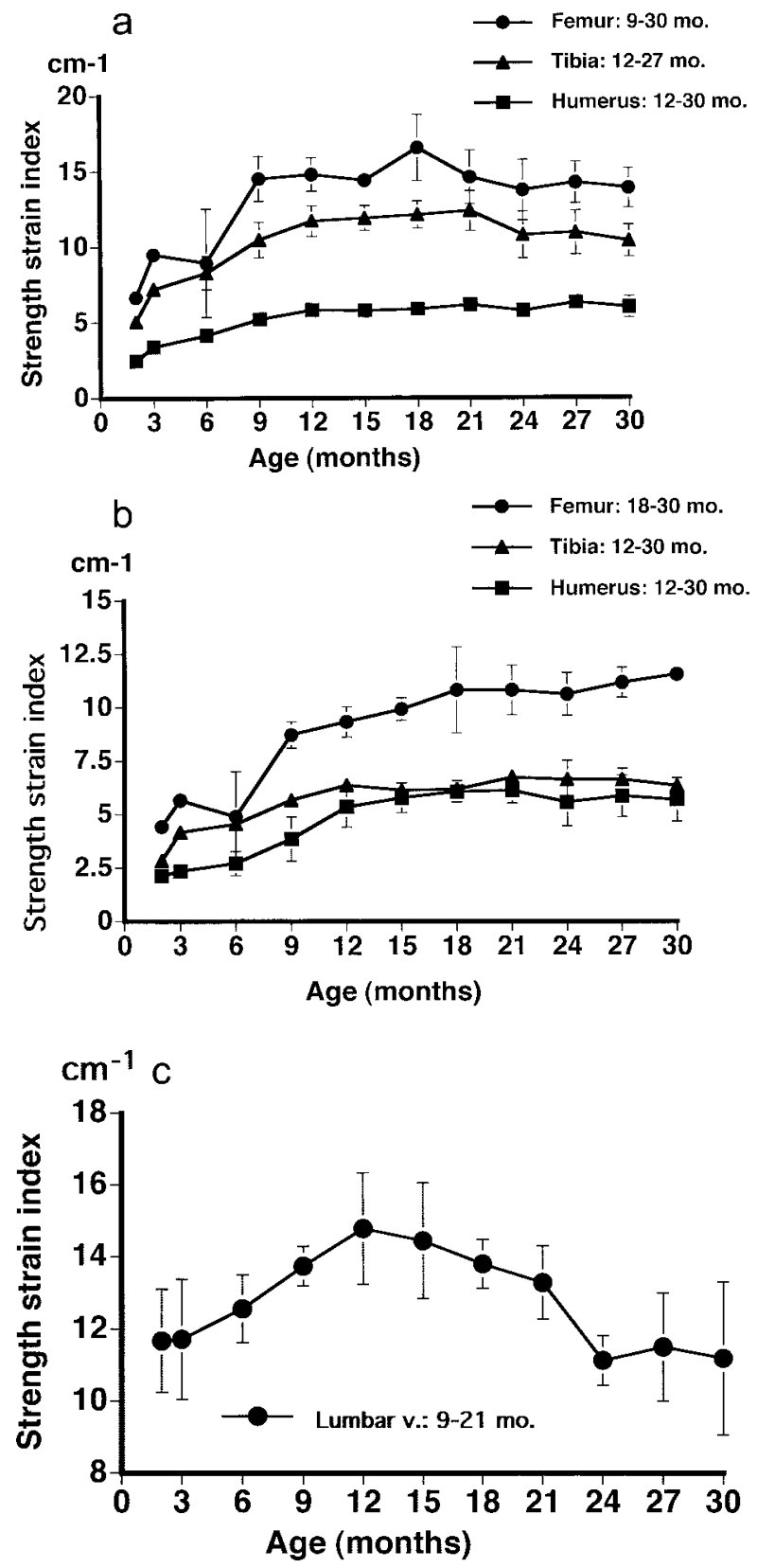

Fig. 7. SSI of the metaphysis (a) and diaphysis (b) of femur, tibia and humerus, and the central cross-section of the first lumbar vertebra (c). The ages showing high values are presented in each figure. Values and bar are mean \pm SD.

differs from that in men in whom BMD decreases with increasing age.

In the present study, it was determined that the total area of long bones and lumbar vertebra continued to enlarge with increasing age. The cortical area decreased while the trabecular area increased with increasing age. This might be due to the dominance of periosteal formation rather than endosteal resorption by the modeling pattern in rats, indicat- 
ing that the cortical thickness thins with increasing age. The greater increase in the SSI values of long bones and the change in first lumbar vertebra might be due to these morphological changes. This might be related to almost no change in organic components with increasing age $[11,13-$ 14].

The age when rats show the so-called peak of bone mass in the whole skeleton was not clearly determined from the results obtained in this study. However, an age-related pattern similar to that observed in humans was found in some bones and parameters by the pQCT method in rats showing the modeling pattern.

\section{REFERENCES}

1. Aitken, J. M., Armstrong, E. and Anderson, J. B. 1972. Osteoporosis after oophorectomy in the mature female rat and the effect of oestrogen and/or progestogen replacement therapy in its prevention. J. Endocrinol. 55: 79-87.

2. Bak, B. and Jensen, K. S. 1992. Standardization of tibial fracture in the rat. Bone 13: 289-295.

3. Bagi, C. M., Wilkie, D., Georgelos, K., Williams, D. and Bertolini, D. 1997. Morphological and structural chracteristics of the proximal frmur in human and rat. Bone 21: 261-267.

4. Baldock, P. A. J., Moore, R.J., Durbridge, T. C. and Morris, H. A. 1990. Comparison of three methods for estimation of bone resorption following ovariectomy in the distal femur and the proximal tibia of the rat. Bone 24: 597-602.

5. Epstein, S., Bryce, G., Hinman, J. W., Miller, O. N., Riggs, B. L., Hui, S. L. and Johnston, C. C. 1986. The influence of age on bone mineral regulating hormones. Bone 7: 421-425.

6. Frost, H. M. and Jee, W. S. S. 1992. On the rat model of human osteopenias and osteoporoses. Bone and Mineral 18: 227-236.

7. Frost, H. M. 1973. pp. 28-53. In: Bone Remodeling and Its Relationship to Metabolic Bone Disease. Charles C Thomas: Springfield.

8. Fukuda S, Matsuoka O. 1979. Maturation process of secondary ossification centers in the rat and assessment of bone age. Exp Anim. (Tokyo) 28: 1-9.

9. Fukuda, S. and Iida, H. 1991. Age and sex-related changes in bone metabolism in normal rats and the effects in ovariectomized and orchidectomized rats at different age. J. Jpn. Soc. Bone Morphom. 1: 89-94.

10. Gasser, J. A. 1995. Assessing bone quantity by pQCT. Bone 17: $145 \mathrm{~S}-154 \mathrm{~S}$.
11. Iida, H. and Fukuda, S. 1993. Age-related changes in bone weights and their components in rats. Exp. Anim. (Tokyo) 42: 349-356.

12. Jee, W. S. S., Li, X. J. and Ke, H. Z. 1991. The skeletal adaptation to mechanical usage in the rat. Cells and Mater. (Suppl) 1: 131-142.

13. Juhn, A., Weiss, A., Mendes, D. and Silbermann, M. 1991. Non-invasive assessment of bone mineral density during maturation and aging of wistar female rats. Cells Mater. (Suppl.) 1: 19-24.

14. Kalu, D. N., Hardin, R. H., Cockerham, R. and Yu, B. P. 1984. Aging and dietary modulation of rat skeleton and parathyroid hormone. Endocrinology 115: 1239-1247.

15. Kalu, D. N., Liu, C. C., Salerno, E., Hollis, B., Echon, R. and Ray, M. 1991. Skeletal response of ovariectomized rats to low and high dose of 17 $\beta$-estradiol. Bone Mineral 14: 175-187.

16. Miller, S. C., Bowman, B. M. and Jee, W. S. S. 1995. Available animal models of osteopenia-small and large. Bone 17: 117S-123S.

17. Schapira, D., Lotan-Miller, R., Barzilai, D. and Silbermann, M. 1991. The rat as a model for studies of the aging skeleton. Cells Mater. (Suppl.) 1: 181-188.

18. Schoutens, A., Verhas, M., L'Hermite-Baleriaux, M., L'Hermite, M., Verschaeren, A., Dourov, N., Mone, M., Heilporn, A. and Tricot, A. 1984. Growth and bone haemodynamic responses to castration in male rats. Reversibility by testosterone. Acta Endocrinol. 107: 428-432.

19. Sontag, W. 1986. Quantitative measurements of periosteal and cortical-endosteal bone formation and resorption in the midshaft of male rat femur. Bone 7: 63-70.

20. Sontag, W. 1992. Age-dependent morphometric alterations in the distal femora of male and female rats. Bone 13: 297-310.

21. Thompson, D. D., Simmons, H. A., Pirie, C. M. and Ke, H. Z. 1995. FDA guidelines and animal models for osteoporosis. Bone 17: 125S-133S.

22. Verhas, M., Schoutens, A., L'hermite-Baleriaux, M., Dourov, N., Verschaeren, A., Mone, M. and Heilporn, A. 1986. The effects of orchidectomy on bone metabolism in aging rats. Calcif. Tissue Int. 39: 74-77.

23. Wink, G. and Felts, W. 1980. Effects of castration on the bone structure of male rats, a model of osteoporosis. Calcif. Tissue Int. 32: 77-82.

24. Wronski, T. J., Schenck, P. A., Cintron, M, and Walsh C. C. 1987. Effect of body weight on osteopenia in ovariectomized rats. Calcif. Tissue Int. 40: 155-159. 